\title{
USING SIMULATION TO VERIFY LIFE HISTORY RELATIONS INDICATED BY TIME SERIES ANALYSIS
}

\author{
A. L. JENSEN* \\ School of Natural Resources, University of Michigan, Ann Arbor, MI 48109-1115, USA
}

\begin{abstract}
SUMMARY
Time series analysis of population abundance is not based on assumptions about the dynamics of populations, but sometimes the results can be interpreted biologically. At other times the results are difficult to interpret. To better understand the results of a time series analysis of a walleye fish population, as it related to the walleye's life history, I compared a time series analysis of walleye field data with a time series analysis of simulated data from a population dynamics model. In the simulations, the nature of the time lags could be identified by changing model parameters. The simulations indicated that a partial autocorrelation coefficient (PAC) at lag 1 would result from density dependence, that a PAC at lag 5 would result from the time required for maturation, and that a negative sign at lag 5 would result from high larval survival. The simulation results help in the interpretation of the PACs obtained in the time series analysis of field data. Copyright (C) 1999 John Wiley \& Sons, Ltd.
\end{abstract}

KEY WORDS walleye; time series; autocorrelation; Great Lakes; simulation

\section{INTRODUCTION}

Autoregressive models are time series models in which future values of a variable are expressed in terms of past values of the variable. Autoregressive models are useful for analysis of population data because future population abundances often are related to past abundances and time lags identified in autoregressive models often are biologically meaningful (e.g. Moran 1953; Jensen 1976, 1985; Liu and Jensen 1992). However, autoregressive models are empirical statistical models that are not based on assumptions about the dynamics of animal populations, and sometimes autoregressive models have been difficult to interpret biologically (e.g. Moran 1953; Jensen 1985; Tong 1990). Simulation studies have been applied to study the source of observed time lags in autoregressive models (e.g. Tong 1990).

In this paper, I used a simulated walleye population to investigate the results of a time series analysis of the Red Lakes, Minnesota, walleye population for which there was a negative partial autocorrelation coefficient (PAC), perhaps corresponding to age at maturity. Time lags in data generated with the simulation model were manipulated by changing model parameters, and time lags similar to those observed in the Red Lakes walleye population were generated. Further analyses of the simulation results identified the ecological process that may have produced the observed time lags in the Red Lakes walleye data.

* Correspondence to: A. L. Jensen, School of Natural Resources, University of Michigan, Ann Arbour, MI 48109-1115, USA.

CCC 1180-4009/99/030237-09\$17.50

Copyright (C) 1999 John Wiley \& Sons, Ltd.

Received 10 December 1997

Accepted 1 December 1998 


\section{RED LAKES WALLEYE POPULATION}

The walleye data were catch (in $1000 \mathrm{~kg}$ ) for the upper and lower Red Lakes fishery in Minnesota from 1930 to 1975 (Smith and Pycha 1961; Smith 1977). The walleye data exhibit fluctuations typical of many fish populations (Figure 1(A)), where occasional large age classes pass through the population and produce high abundances for several years followed by lower abundances (Smith 1977). In the partial autocorrelation function (PACF) for the Red Lakes walleye there is a large partial autocorrelation coefficient of 0.55 at lag 1 that is statistically significant and a smaller PAC of -0.25 at lag 5 that is not quite statistically significant (Figure 1(B)). This same pattern occurs with other walleye populations and other fish species (e.g. Jensen 1985; Liu and Jensen 1992; Carlander and Payne 1977).

A first order autoregressive model was fitted to the walleye data giving

$$
Y_{t+1}=132 \cdot 35+0 \cdot 57 Y_{t}
$$
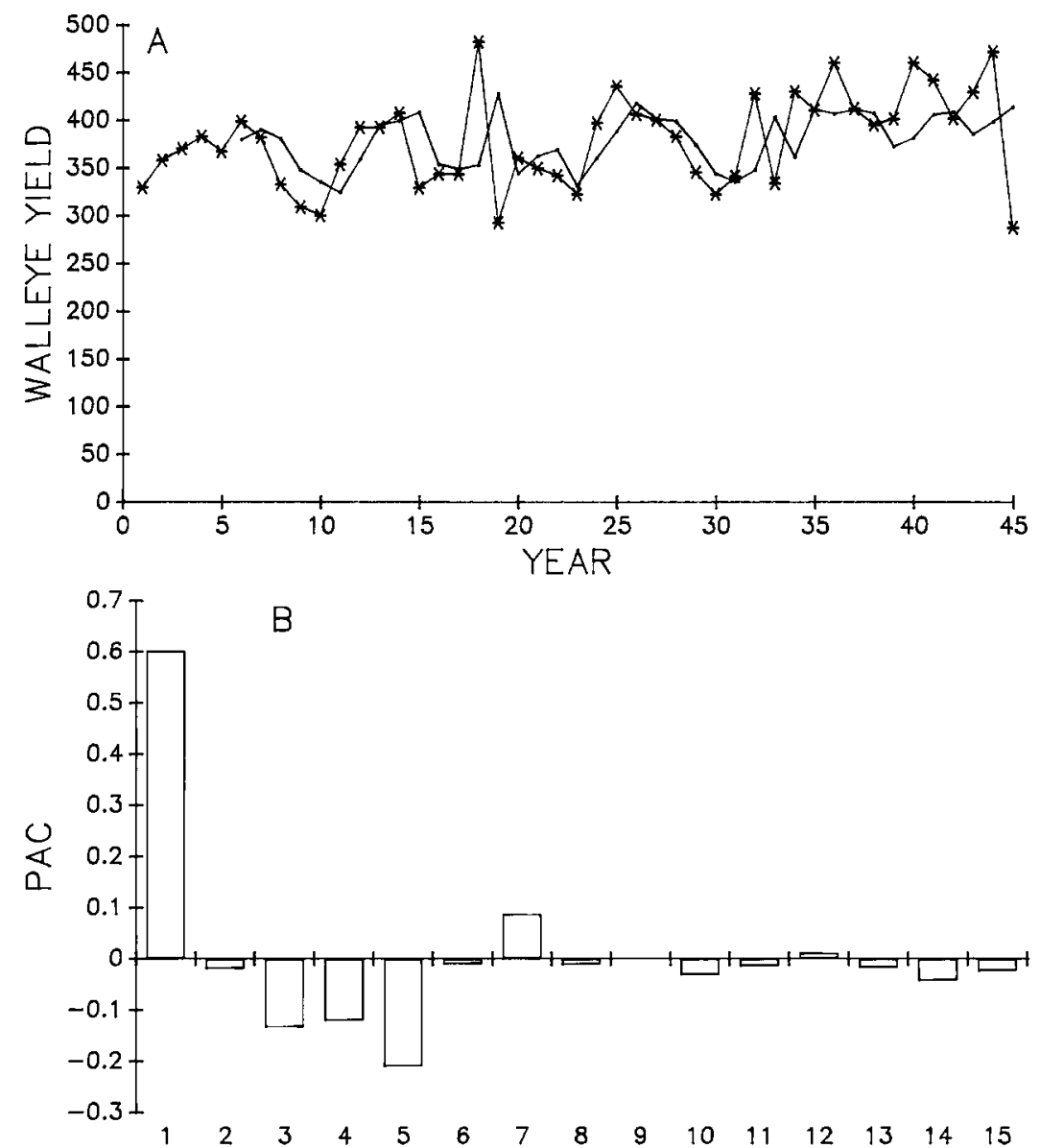

Figure 1. (A) Observed walleye catch $(1000 \mathrm{~kg})$ for the Red Lakes, Minnesota, fishery and values predicted with the AR(1) forecasting equation. (B) Partial autocorrelation function for the Red Lakes walleye fishery 
with $R^{2}=0 \cdot 31$, where $Y_{t}$ is catch (in $1000 \mathrm{~kg}$ ) at time $t$. Equation (1) accounts for a fair amount of the variation in walleye yield and provides reasonable one-year ahead forecasts (Figure 1(A)). The PAC at lag 5 was not statistically significant (Figure 1(B)), but the length of the data series was short $(n=45)$, and the PAC at lag 5 might represent a biologically meaningful reproductive time lag because the age at maturity of female walleye in the Red Lakes was age 5 (Smith and Pycha 1961). Walleye data reported by Carlander and Payne (1977) for Clear Lake, Iowa, indicate a nearly significant negative PAC at lag 4 and walleye matured at age 4 in Clear Lake. The reason for the negative PACs is not clear.

\section{METHODS}

A simulation model for walleye populations was used to verify the biological significance of the PACs identified in the analysis of the Red Lakes data. The simulation model was discussed in detail by Jensen $(1989,1991)$. The model combines results from Larkin's (1966) predator-prey model, Ursin's (1967) bioenergetic growth model, Ivlev's (1961) feeding model, the exponential mortality model, and the relation between mortality and size proposed by Peterson and Wroblewski (1984). The model tracks the number of individuals $(N)$ and the mean weight of an individual in the larval, YOY, and juvenile lifestages, and in 14 age classes of adults. Based on Winberg's (1956) energy balance equation, Ursin (1967) proposed modelling growth as

$$
\mathrm{d} W / \mathrm{d} x=C\left\{1-\exp \left[-S_{\mathrm{f}}\left(K_{i}-g_{i} N_{2}\right)\right]\right\} W^{m}-k W^{n}
$$

where $C, k, n$ and $m$ are metabolic parameters, $K_{i}$ is prey carrying capacity $(i=1,2,3,4$ for larvae, YOY, juveniles and adults), $g_{i}$ measures the impact of the predator on prey abundance ( $i-1,2,3,4$ for larvae, YOY, juveniles and adults), $N_{2}$ is walleye abundance, and $s_{\mathrm{f}}$ is a feeding parameter (Ursin 1967). Reproduction occurs annually, with the number of eggs produced given by

$$
E(t)=H \sum_{x=x_{\mathrm{m}}}^{x_{\mathrm{v}}} N(x, t) W(x, t) / 2
$$

where $H$ is the average number of eggs per gram of female, $x_{\mathrm{m}}$ is age at maturity, $x_{\mathrm{v}}$ is the oldest age attainable, $N(x, t)$ is abundance of age $x$ individuals in the population at time $t$, and $W(x, t)$ is the mass of age $x$ individuals at time $t$. After reproduction the sizes of all reproducing adults were reduced by the amounts $H W(0) W(x, t)$, where $W(0)$ was egg mass.

The number of larvae hatching after the egg incubation period was $s \times E(t)$, where $s$ was the egg survival rate. Mortality after hatching was modeled with the exponential model

$$
\mathrm{d} N(x, t) / \mathrm{d} t=-Z N(x, t)
$$

where $N(x, t)$ was abundance of age $x$ at time $t$ and $Z$ was the instantaneous mortality coefficient for ages $\geqslant x_{r}$. $Z$ consisted of fishing $\mathrm{F}$ and natural mortality $\mathrm{M}$. Natural mortality was related to fish size using the equation of Peterson and Wroblewski (1984)

$$
M=a W(x, t)^{-b}
$$


where $a$ and $b$ were parameters. Observed mortality of larvae was considerably higher than that calculated with the Peterson and Wroblewski (1984) equation. McGurk (1986) hypothesized that the higher larval mortality was related to spatial patchiness of larvae, and he obtained reasonable values for mortality when Peterson and Wroblewski (1984) estimates were multiplied with Loyd's index of patchiness. I applied an approach similar to that of McGurk (1986) and multiplied Peterson and Wroblewski (1984) estimates with a parameter $p$. Random variation in abundance was obtained by adding to $p$ a normally distributed variable with mean zero and standard deviation $0 \cdot 20$.

Duration of the larval and YOY life stages were relatively short at 0.0822 and 0.75 years, so for these life stages the model was solved by replacing the differential equations with difference equations. For juveniles and adults the equations were solved using Euler's method with a time interval of $0 \cdot 1$ years. The model was applied using data for the walleye population of the western basin of Lake Erie, and the parameter estimates are the same as in earlier studies where the sources of the estimates are given (Jensen 1989, 1991). Estimates of the model parameters are summarised in Table I.

Simulations of 500 years were done with two different levels of larval survival and ages at maturity of 4 and 5 years. Larval survival was manipulated by changing the coefficient of patchiness $p$; a high level of survival was obtained with $p=5.50$ and a low level of survival was obtained with $p=6.75$. The first 50 years for each simulation were discarded to minimize the effects of initial conditions, and the PACFs were calculated using data for years 51-500.

Table I. Summary of simulation model parameter estimates

\begin{tabular}{llc}
\hline Parameter & Identification & Value \\
\hline$W_{0}$ & Initial weight (g) & $0 \cdot 0025$ \\
$h$ & Number of eggs per gram of female & 74 \\
$x_{\mathrm{m}}$ & Age at maturity (years) & 4 \\
$x_{\mathrm{v}}$ & Oldest age attainable (years) & 18 \\
$x_{\mathrm{c}}$ & Age at entry into fishery (years) & 4 \\
$F$ & Instantaneous fishing mortality (year $\left.{ }^{-1}\right)$ & $0 \cdot 25$ \\
$p$ & Coefficient of patchiness & $6 \cdot 50$ \\
$K_{1}$ & Carrying capacity of larval food $\left(\mathrm{mg} / \mathrm{m}^{-3}\right)$ & 200 \\
$K_{i}$ & Carrying capacity of YOY, juvenile, and adult food $\left(\mathrm{mg} / \mathrm{m}^{3}\right) ; i=2,3,4$ & 2000 \\
$g_{1}$ & Effect of larval on their food & $1 \times 10^{-6}$ \\
$g_{2}$ & Effect of YOY on their food & $3 \times 10^{-3}$ \\
$g_{3}$ & Effect of juveniles on their food & $4 \times 10^{-2}$ \\
$g_{4}$ & Effect of adults on their food & $9 \times 10^{-2}$ \\
$C$ & Metabolic coefficient & 15 \\
$k$ & Metabolic coefficient & $0 \cdot 60$ \\
$m$ & Metabolic exponent & $0 \cdot 66$ \\
$n$ & Metabolic exponent & 1 \\
$s_{\mathrm{f}}$ & Coefficient in food relation & $0 \cdot 004$ \\
$s$ & Egg survival (year $\left.{ }^{-1}\right)$ & $0 \cdot 50$ \\
$a$ & Coefficient in Peterson-Wroblewski equation & $1 \cdot 92$ \\
$b$ & Exponent in Peterson-Wroblewski equation & $0 \cdot 25$ \\
\hline
\end{tabular}




\section{RESULTS AND DISCUSSION}

Changing age at maturity in the simulations changed the lag at which a significant reproductive PAC occurred in the yield data, and changing the coefficient of patchiness $p$, which changed survival of larvae, changed the sign of the reproductive PAC. If age at maturity was 4 , a significant PAC occurred at lag 4 (Figures 2(B) and 3(B)) and if age at maturity was 5, a significant PAC occurred at lag 5 (Figures 4(B) and 5(B)). If larval survival was high $(p=5 \cdot 5)$, the reproductive PAC was negative (Figures $2(B)$ and $4(B)$ ), and if larval survival was low $(p=6 \cdot 75)$, the reproductive PAC was positive (Figures 3(B) and 5(B)). Hence, the PAC at lag 5 in the Red Lakes walleye data could be a reproductive time lag, and the negative sign would indicate that larval survival in the Red Lakes was high.

To identify the process that led to the positive or negative sign of the PAC at the reproductive lag in the simulations, I examined the state variables of the model at year 200 (Table II). The state variables indicate that when larval survival was high $(p=5 \cdot 5$, Cases 1 and 3$)$, the number of
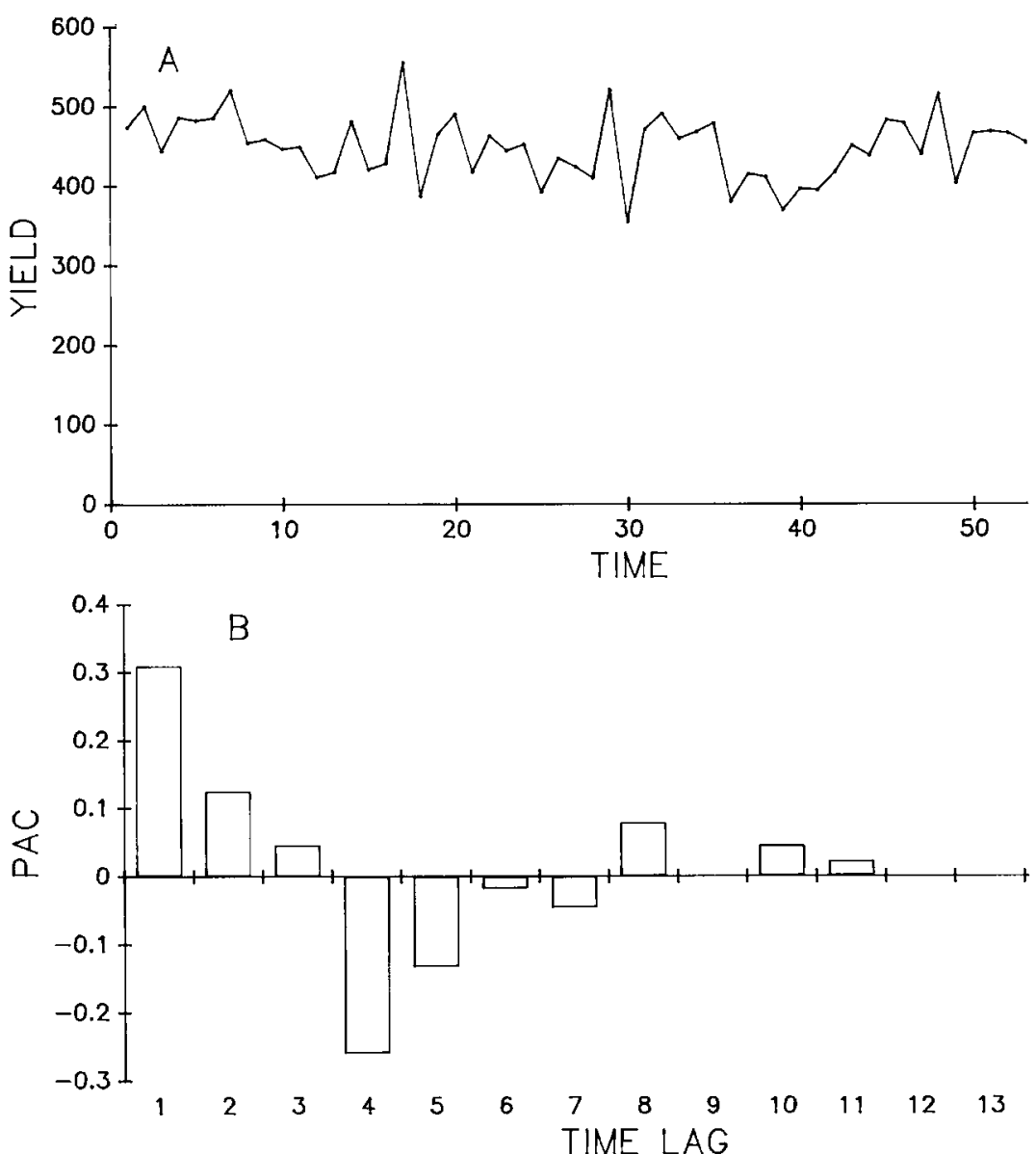

Figure 2. (A) Simulated walleye yield (1000 kg) with high larval survival and maturity at age 4. (B) Partial autocorrelation function with high larval survival and maturity at age 4 

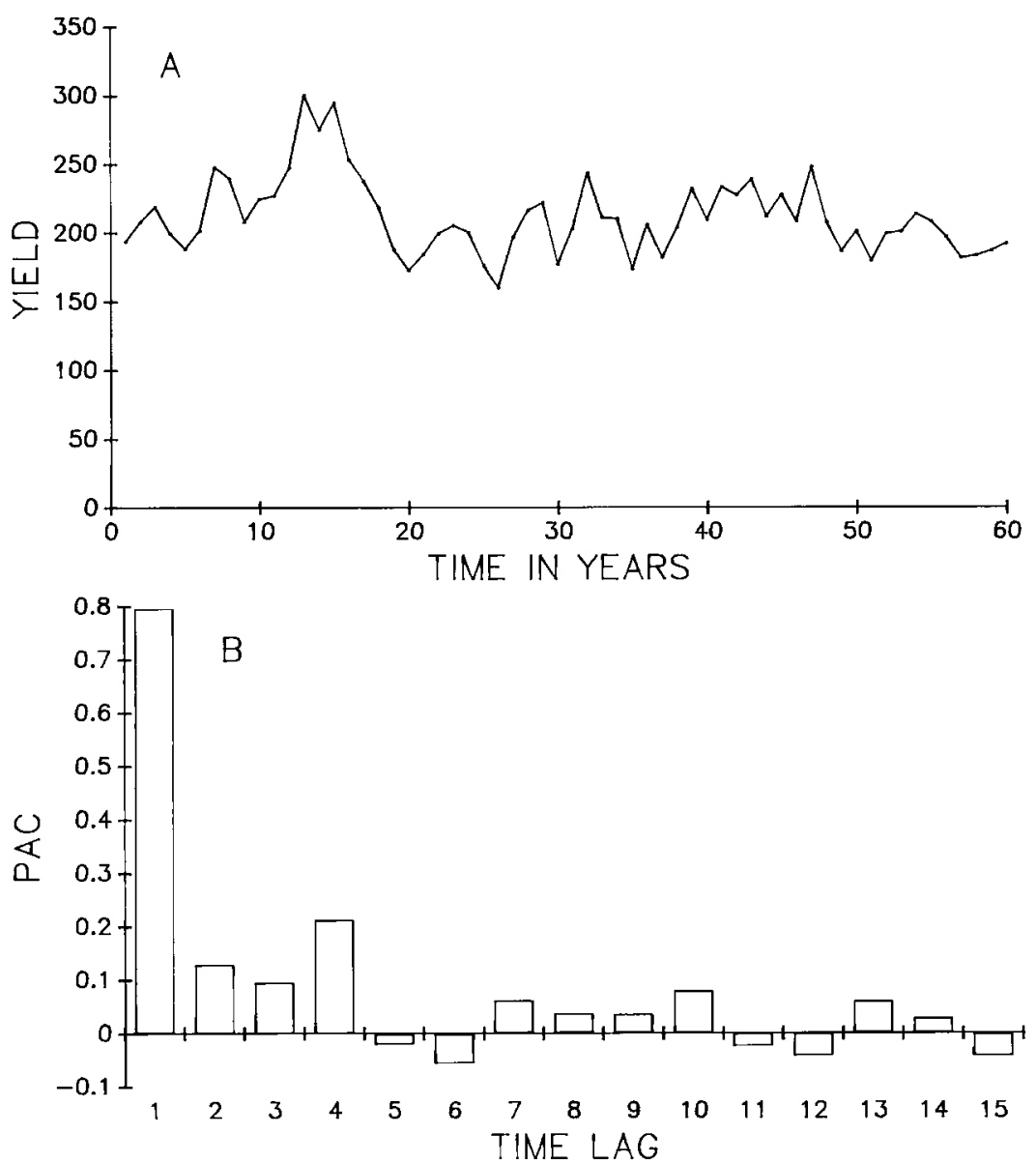

Figure 3. (A) Simulated walleye yield (1000 kg) with low larval survival and maturity at age 4. (B) Partial autocorrelation function with low larval survival and maturity age 4

recruits to maturity was high, adult biomass was large, the number of eggs produced was large, the growth coefficient was low, the asymptotic weight was high, the inflection point in the growth curve occurred at an older age, and food concentrations available to YOY and juveniles were lower. The above results indicated that when larval survival was high, population densities were high and food resources were limiting for YOY and juveniles, which led to slow growth of YOY and juveniles (Table II). Thus, when there was a large population of spawners which produced many eggs, the high abundance of young resulted in even slower growth and higher mortality for YOY and juveniles and a lower recruitment to mature adults 4 or 5 years later. This resulted in a negative reproductive PAC. On the other hand, when larval survival was low, population densities were low, food resources were not limiting, and YOY and juveniles grew quickly. If a large population of spawners produced a large number of eggs, the eggs that survived the larval stage had a high growth and survival as YOY and juveniles, which resulted in a relatively large recruitment and thus a positive reproductive PAC. 

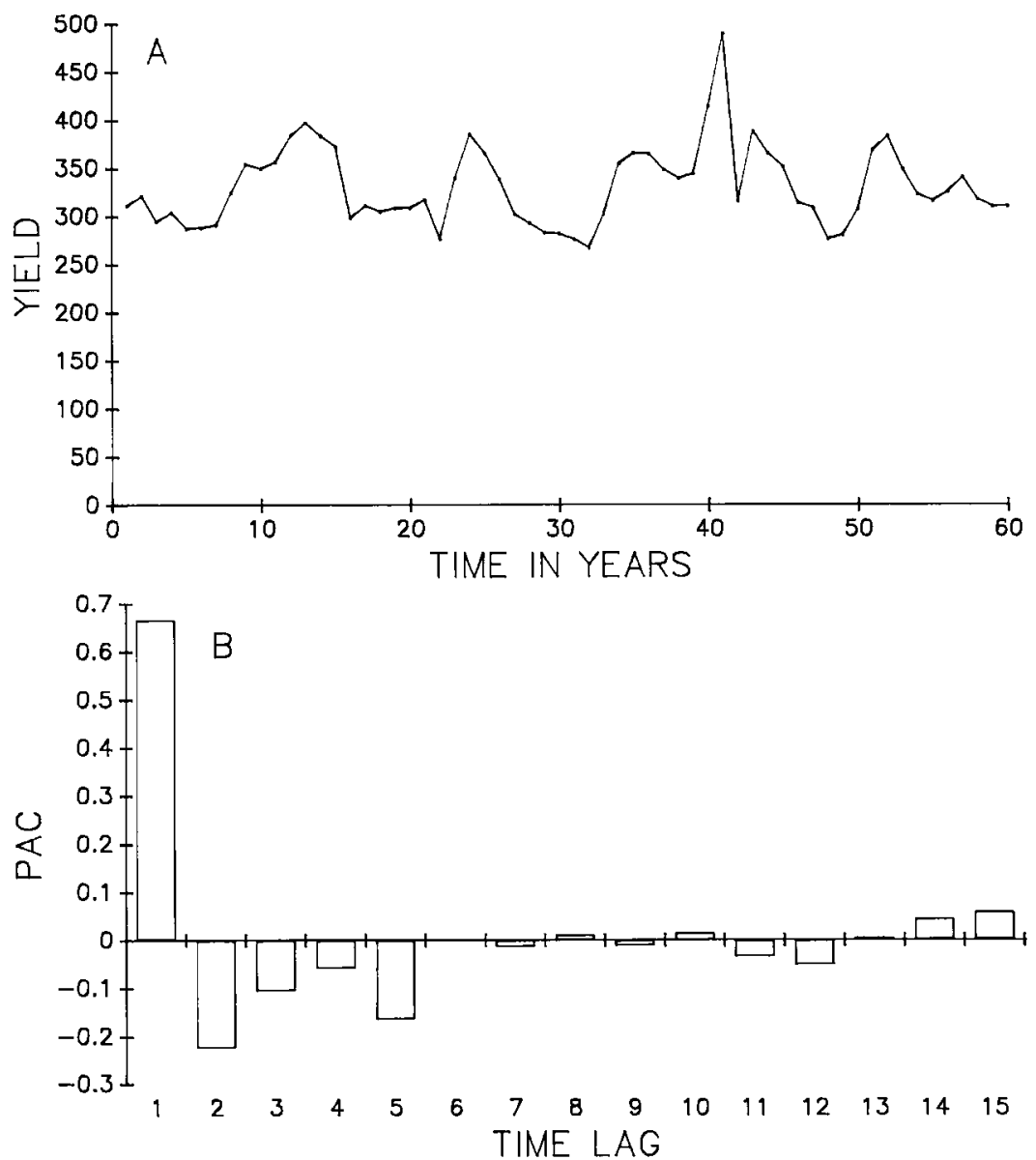

Figure 4. (A) Simulated walleye yield (1000 kg) with high larval survival and maturity at age 5. (B) Partial autocorrelation function with high larval survival and maturity at age 5

The large PAC at age 1 in the Red Lakes data and in all of the simulations, indicated density dependent population growth, which can be interpreted in terms of logistic growth about a carrying capacity. If the logistic equation is linearized near its carrying capacity using a Taylor series expansion, the difference equation form of the resulting equation for population growth is

$$
N_{t+1}=r K+(1-r) N_{t}
$$

where $r$ is the intrinsic rate of increase and $K$ is the carrying capacity. Equation (6) is the same as the first order autoregressive model

$$
N_{t+1}=(1-\phi) \mu+\phi N_{t}+\mu_{t}
$$

with $K=\mu$ and $r=1-\phi$ where $\mu$ is the mean of the series, $\phi$ is the PAC for lag 1 , and $\mu_{t}$ is a random shock. Thus, data that can be described with a first order autoregressive model also can be described with the logistic equation. In the simulations, population growth was logistic 

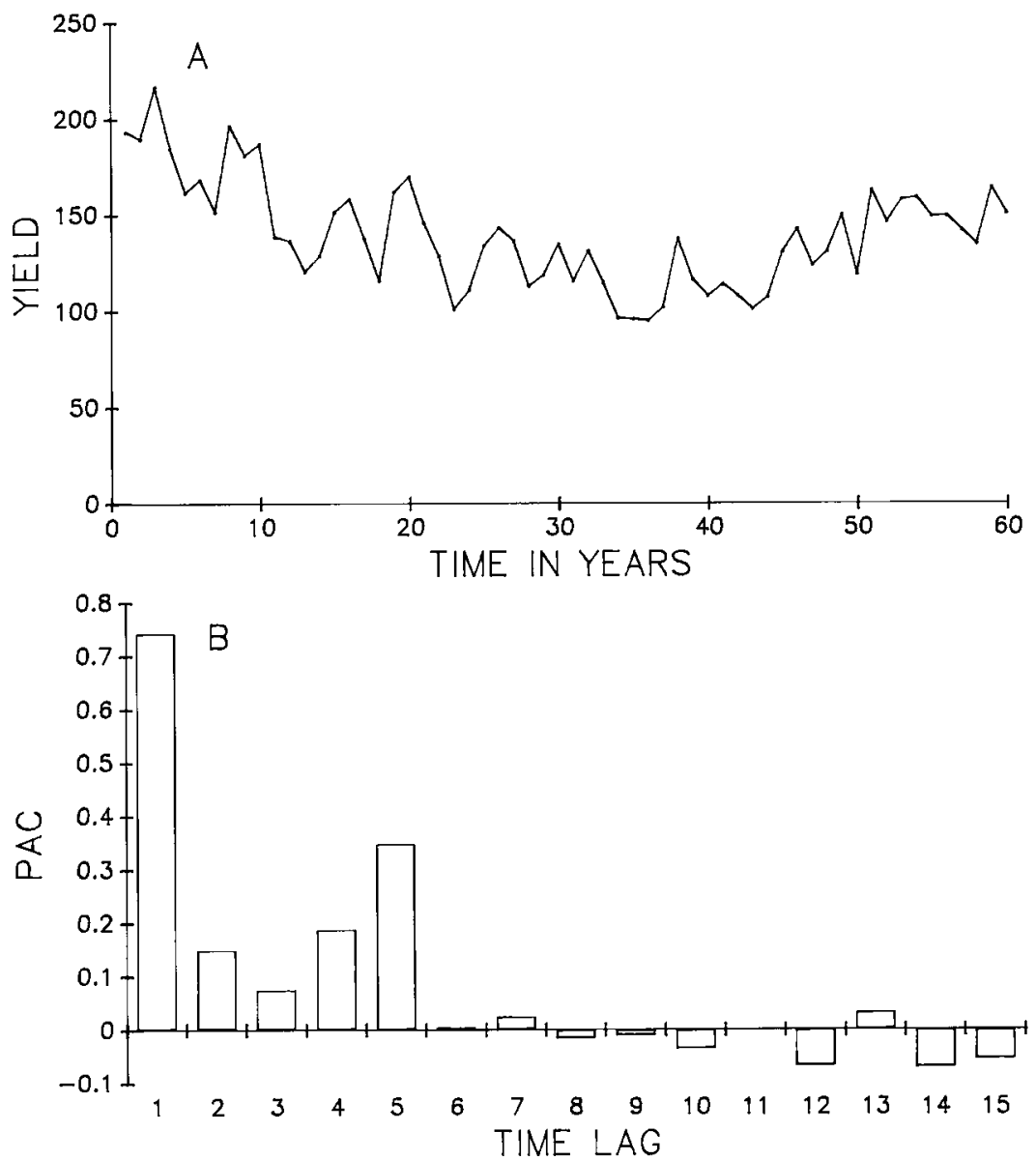

Figure 5. (A) Simulated walleye yield (1000 kg) with low larval survival and maturity at age 5. (B) Partial autocorrelation function with low larval survival and maturity at age 5

because there were 14 adult age groups in the simulated walleye population which averaged out variation in recruitment from year to year so that abundance of the population in one year was related mainly to abundance in the past year; population size changed gradually from year to year and the best predictor of abundance at year $t$ was abundance at year $t-1$.

In summary, time lags similar to those observed in the Red Lakes walleye catch data were generated with the simulation model. Although the simulation results cannot confirm the source of the time lags in the Red Lakes walleye, the simulations indicated that a PAC at lag 1 would result from density dependence, a PAC at lag 5 would result from the time required for maturation, and a negative sign at lag 5 would result from a high larval survival.

\section{ACKNOWLEDGEMENTS}

I thank Kenneth Rose for many helpful editorial comments. 
Table II. State variables at year 200. For case $1, x_{\mathrm{m}}=4$ and $p=5 \cdot 5$; for case $2, x_{\mathrm{m}}=4$ and $p=6 \cdot 75$; for case $3, x_{\mathrm{m}}=5$ and $p=5 \cdot 5$; and for case $4, x_{\mathrm{m}}=5$ and $p=6 \cdot 75$

\begin{tabular}{lcccc}
\hline Variable & Case 1 & Case 2 & Case 3 & Case 4 \\
\hline Recruits & 883 & 228 & 967 & 100 \\
Biomass $(\mathrm{kg})$ & 2513 & 918 & 1236 & 336 \\
Eggs & $7 \cdot 71 \times 10^{7}$ & $3 \cdot 09 \times 10^{7}$ & $5 \cdot 08 \times 10^{7}$ & $1 \cdot 18 \times 10^{7}$ \\
$K\left(\right.$ year $\left.^{-1}\right)$ & $0 \cdot 21$ & $0 \cdot 29$ & $0 \cdot 15$ & $0 \cdot 30$ \\
$W_{\text {inf }}(\mathrm{g})$ & 6189 & 5648 & 7774 & 5787 \\
Inflection $^{\mathrm{a}}$ & $5 \cdot 30$ & $3 \cdot 78$ & $7 \cdot 33$ & $3 \cdot 69$ \\
$M\left(\right.$ year $\left.^{-1}\right)$ & $0 \cdot 43$ & $0 \cdot 43$ & $0 \cdot 37$ & $0 \cdot 39$ \\
Larval $^{\mathrm{b}}$ & 161 & 184 & 174 & 194 \\
YOY $^{\mathrm{b}}$ & 422 & 936 & 387 & 978 \\
Juvenile $^{\mathrm{b}}$ & 415 & 889 & 224 & 938 \\
Adults $^{\mathrm{b}}$ & 874 & 968 & 938 & 991 \\
\hline
\end{tabular}

${ }^{a}$ Age in years at inflection of the growth curve.

${ }^{b}$ Food concentrations in $\mathrm{mg} \mathrm{m}^{-3}$ for Larval, YOY, juvenile and adults.

\section{REFERENCES}

Carlander, K. D. and Payne, P. M. (1977). 'Year-class abundance, population and production of walleye (Stizostedion vitreum vitreum) in Clear Lake, Iowa, 1948-74, with varied fry stocking rates'. Journal of the Fisheries Research Board of Canada 34, 1792-1799.

Ivlev, V. S. (1961). The Experimental Ecology of the Feeding of Fish. New Haven: Yale University.

Jensen, A. L. (1976). 'Time series analysis and forecasting of Atlantic menhaden catch'. Chesapeake Science 17, 305-307.

Jensen, A. L. (1985). 'Time series analysis and forecasting of menhaden catch and CPUE'. North American Journal of Fisheries Management 5, 78-85.

Jensen, A. L. (1989). 'Simulation of the potential for life history components to regulate walleye population size'. Ecological Modelling 45, 27-41.

Jensen, A. L. (1991). 'Simulation of fish population responses to exploitation'. Ecol. Model. 55, $203-218$.

Larkin, P. A. P. (1966). 'Exploitation in a type of predator-prey relationship'. Journal of the Fisheries Research Board of Canada 23, 349-356.

Liu, K. M. and Jensen, A. L. (1992). 'Validation of lake whitefish catch per unit effort data using time series and cross correlation analysis'. Trans. Amer. Fish Soc. 121, 797-801.

McGurk, M. D. (1986). 'Natural mortality of marine pelagic fish eggs and larvae: role of spatial patchiness'. Marine Ecology Prog Series 34, 227-242.

Moran, P. A. P. (1953). 'The statistical analysis of the Canadian lynx cycle. I. Structure and prediction'. Australian Journal of Zoology 1, 163-173.

Peterson, I. and Wroblewski, J. S. (1984). 'Mortality rates of fishes in the pelagic ecosystem'. Canadian Journal of Fisheries and Aquatic Sciences 41, 1117-1120.

Smith, L. L. Jr (1977). 'Walleye (Stizostedion vitreum vitreum) and yellow perch (Perca flavescens) populations and fisheries of the Red Lakes, Minnesota, 1930-75'. J. Fish. Res. Board Can. 34, 1774-1783.

Smith, L. L. Jr and Pycha, R. L. (1961). 'Factors related to commercial production of the walleye in Red Lakes, Minnesota'. Trans. Amer. Fish. Soc. 90, 190-217.

Tong, H. (1990). Non-linear Time Series. New York: Oxford University Press.

Ursin, E. (1967). 'A mathematical model of some aspects of fish growth, respiration, and mortality'. Journal of the Fisheries Research Board of Canada 24, 2355-2390.

Winberg, G. G. (1956). 'Rate of metabolism and food requirements in fishes'. English translation. Fisheries Research Board of Canada. Transl. No. 194, 1960. 